\title{
Queda transiente de uma partícula esférica num fluido viscoso: Transformação da equação íntegro-diferencial em uma equação diferencial
}

\author{
Antônio Santos Silva \\ Universidade Federal de Sergipe - Departamento de Matemática \\ 49100-000, Cidade Universitária Prof. José Aloísio de Campos, São Cristóvão, SE \\ E-mail: ass@infonet.com \\ Edilson de Jesus Santos \\ Universidade Federal de Sergipe - Departamento de Engenharia Química \\ 49100-000, Cidade Universitária Prof. José Aloísio de Campos, São Cristóvão, SE \\ E-mail: edilsonjs@ufs.br
}

Resumo: Este trabalho, por meio da transformação de uma equação integro-diferencial em uma equação diferencial, apresenta alternativas para a determinação da velocidade de queda acelerada de uma partícula esférica num fluido newtoniano incompressivel sob o regime de Stokes.

\section{Introdução}

Dos trabalhos de Feng e Joseph (1995), Mollinger et al (1999), Ardekani e Rangel (2006), Soares et al (2012) e Srivastava et al (2013), a modelagem apresentada por Basset (1888) para a velocidade de queda acelerada de uma partícula esférica, inicialmente em repouso, num fluido newtoniano incompressível, sob o regime de Stokes, pode ser usada como ponto de partida para estudo de queda de corpos em fluidos, principalmente quando envolve história da aceleração que torna a equação do movimento uma equação íntegro-diferencial. Este trabalho mostra que a equação íntegro-diferencial, tratada em Basset (1888), pode ser transformada em uma equação diferencial ordinária linear de segunda ordem, com coeficientes constantes, não homogênea, a qual gera alternativas para a determinação da velocidade. Tal transformação abre caminho para estudos de quedas de corpos em fluidos que requerem termos adicionais, no sentido de que, para não envolver história de aceleração, os termos adicionais sejam colocados na equação diferencial de segunda ordem.

\section{Elementos básicos}

De acordo com Basset (1961), a velocidade $V(t)$ da partícula esférica esfera no instante $t$ é obtida das equações

$$
\begin{aligned}
& m \frac{d V(t)}{d t}=(P-E)-R(t) \quad m=\frac{4}{3} \pi a^{3} \rho_{s} \quad P-E=\frac{4 \pi a^{3}}{3}\left(\rho_{s}-\rho\right) g \\
& R(t)=\frac{2}{5} \pi a^{3} \rho V^{\prime}(t)+6 \pi \mu a V(t)+6 a^{2} \sqrt{\pi \mu \rho} \int_{0}^{t} \frac{V^{\prime}(\tau)}{\sqrt{t-\tau}} d \tau
\end{aligned}
$$

onde $E$ é o empuxo, $R$ é a resistência dinâmica oferecida pelo fluido ao movimento da partícula esférica de massa $m$, raio $a$ e peso $P, \rho_{s}$ e $\rho$ são as massas específicas da partícula e do fluido, respectivamente, $g$ é a aceleração da gravidade e $\mu$ é a viscosidade do fluido. Em variáveis adimensionais, segue das Equações (1) e (2) que: 
$u^{\prime}(\theta)+u(\theta)+M \int_{0}^{\theta} \frac{u^{\prime}(\theta-\lambda)}{\sqrt{\lambda}} d \lambda=1$

$u=\frac{V}{V_{0}}, \theta=A t, \pi M^{2}=\frac{9 \rho}{2 \rho_{s}+\rho}, M>0$,

$A=\frac{9 \mu}{a^{2}\left(2 \rho_{s}+\rho\right)}, V_{0}=\frac{2 a^{2}\left(\rho_{s}-\rho\right) g}{9 \mu}$,

onde $V_{0}$ é a velocidade limite de Stokes.

\section{Determinação da velocidade de queda}

Da condição de velocidade inicial nula, da regra de Leibniz e da Equações (3), (4) e (5)

$u(\theta)+\int_{0}^{\theta}\left(1+\frac{M}{\sqrt{\lambda}}\right) u(\theta-\lambda) d \lambda=\theta$

$u(0)=0,\left(u^{\prime}(0)=1\right)$,

Seja $F(\theta)$ definida por

$Q(\theta)=u^{\prime \prime}(\theta)+2 r u^{\prime}(\theta)+u(\theta)$

onde $r$ é uma constante a ser escolhida adequadamente. Então, de (6), (7) e a regra de Leibnitz, obtemos a equação

$Q(\theta)+\int_{0}^{\theta}\left(1+\frac{M}{\sqrt{\lambda}}\right) Q(\theta-\lambda) d \lambda=\theta+r-1-\frac{M}{\sqrt{\theta}}$

Como,

$\int_{0}^{\theta}\left(1+\frac{M}{\sqrt{\lambda}}\right)\left(1-\frac{M}{\sqrt{\theta-\lambda}}\right) d \lambda=\theta-\pi M^{2}$,

da Equação (4) a escolha

$2 r=2-\pi M^{2}=\frac{4 \rho_{s}-7 \rho}{2 \rho_{s}+\rho}$

em (8) mostra que

$Q(\theta)=1-\frac{M}{\sqrt{\theta}}$

Logo, das Equações (6) a (11), obtemos que a velocidade de queda é dada pelo problema de valor inicial

$u^{\prime \prime}(\theta)+2 r u^{\prime}(\theta)+u(\theta)=1-\frac{M}{\sqrt{\theta}}, \theta>0$

$u(0)=0, u^{\prime}(0)=1$

Para o movimento de queda da partícula, a condição $\rho<\rho_{s}$, pela Equação(10), implica que

$-0,5<r<1$. 
Da aplicação do método da variação de parâmetros na equação diferencial do problema formado pelas Equações (12) e (13), podemos evidenciar duas funções $F(\theta)$ e $G(\theta)$ definidas por:

$F^{\prime}+r F=-\frac{M}{b} \frac{\operatorname{sen} b \theta}{\sqrt{\theta}}, \theta>0$,

$F(0)=1$,

$G^{\prime}+r G=-\frac{M}{b} \frac{\cos b \theta}{\sqrt{\theta}}, \theta>0$,

$G(0)=\frac{1-r}{b}$

Assim, usando (12), (13), (15) e (16), não é difícil ver por substituição direta que

$u=1-F \cos b \theta+G \operatorname{sen} b \theta$

é a solução do problema (12) e (13) para todo $r$ satisfazendo a relação (14), sendo que as formas de integrações para $F$ e $G$ são determinadas de acordo com o sinal de $r$.

No caso de $r<0$

$F(\theta)=\frac{M}{b} e^{-r \theta} \int_{\theta}^{\infty} \frac{e^{r \lambda} \operatorname{sen} b \lambda}{\sqrt{\lambda}} d \lambda,-0,5<r<0$,
$G(\theta)=\frac{M}{b} e^{-r \theta} \int_{\theta}^{\infty} \frac{e^{r \lambda} \cos b \lambda}{\sqrt{\lambda}} d \lambda,-0,5<r<0$.

Para o caso $r=0$

$F(\theta)=\sqrt{\frac{2}{\pi}} \int_{\theta}^{\infty} \frac{\operatorname{sen} \lambda}{\sqrt{\lambda}} d \lambda, r=0$,

$G(\theta)=\sqrt{\frac{2}{\pi}} \int_{\theta}^{\infty} \frac{\cos \lambda}{\sqrt{\lambda}} d \lambda, r=0$.

Finalmente, para $r>0$

$F(\theta)=e^{-r \theta}\left(1-\frac{M}{b} \int_{0}^{\theta} \frac{e^{r \lambda} \operatorname{sen} b \lambda}{\sqrt{\lambda}} d \lambda\right), 0<r<1$,

$G(\theta)=e^{-r \theta}\left(1-\frac{1-r}{b}-\frac{M}{b} \int_{0}^{\theta} \frac{e^{r \lambda} \cos b \lambda}{\sqrt{\lambda}} d \lambda\right), 0<r<1$.

Como, pelas Equações (18) até (23), os limites

$\lim _{\theta \rightarrow \infty} F(\theta)=\lim _{\theta \rightarrow \infty} G(\theta)=0$

são válidos para todo $r$ definido por (14), então pelas Equações (4) e (24)

$\lim _{\theta \rightarrow \infty} u(\theta)=1, \lim _{t \rightarrow \infty} V(t)=V_{0}$

mostrando que, realmente, a partícula atinge a velocidade limite de Stokes.

Os resultados expressos em (25) e o fato da velocidade ser limitada ainda não completam a análise do movimento de queda, uma vez que a Equação (17) apresenta funções 
oscilantes seno e cosseno, onde, fisicamente, esperam-se que as oscilações sejam compensadas com as de $F(\theta)$ e $G(\theta)$ dadas pelas Equações (15) e (16), respectivamente. Como $F(\theta)$ e $G(\theta)$ são dadas por integrais não diretas, usamos a seguir dois caminhos para resolver a questão das oscilações: um é o cálculo de uma série assintótica para $u(\theta)$ obtida diretamente de (12) e (13) e o outro é a determinação de $u(\theta)$ por (17) através da solução numérica de (15) e (16).

\section{$\underline{\text { Solução em série assintótica }}$}

Como, no sistema CGS, o valor de $A$ em geral é grande, a variável $\theta$ definida em (4) assume valores, relativamente altos para valores pequenos de $t$. Isso motiva a solução de (12) e (13) em série assintótica, no lugar da série de potências em torno de $\theta=0$. Por causa disso e do termo não homogêneo de (12), introduzimos a variável $Z$ definida por:

$Z=\frac{1}{\sqrt{\theta}}$

Então, a substituição em (12) de uma série de potências para $u(\theta)$ em torno de $Z=0$ fornece o resultado

$u=1-\sum_{n=0}^{\infty} u_{n} Z^{2 n+1}$

onde os coeficientes $u_{n}$ são determinados das relações

$u_{0}=M, u_{1}=r M$,

$u_{n}=(2 n-1)\left(r u_{n-1}-\frac{2 n-3}{4} u_{n-2}\right)$,

para $n \geq 2, n$ inteiro.

Vale ressaltar que o conjunto de Equações (26) a (29) de imediato mostra que, para $\theta$ suficientemente grande, $u(\theta)$ é aproximadamente dada por

$u(\theta) \simeq 1-\frac{M}{\sqrt{\theta}}$

com erro proporcional a $Z^{3}$, onde $Z$ é dada por (26).

\section{$\underline{\text { Solução numérica }}$}

Para a solução numérica de (15) e (16), adotamos o método de Runge-Kutta de quarta ordem. Como o termo não homogêneo de (16) apresenta um polo em $\theta=0$, em (16) fazemos a mudança de função definida por

$G(\theta)=S(\theta)+\frac{2 M}{b} e^{-\sqrt{\theta}}, 0 \leq \theta<\infty$.

\section{Resultados}

Objetivando a análise da velocidade de queda para cada sinal de $r$, foram usados os valores $r=-0,25, r=0$ e $r=0,50$ em acordo com (14).

Com relação ao cálculo de $u(\theta)$ por (17) e (31) através da solução numérica de (15), (16) e (31), pelo método Runge-Kutta de quarta ordem já citado, foi verificado que para $r=-0,25$ a velocidade calculada oscila para $\theta>23,50$ como o passo de 0,01 . Mas, com o 
passo de 0,0001 oscila para $\theta>48,60$, mostrando que as oscilações não são da velocidade de queda. Elas são oriundas da instabilidade do método numérico para passos maiores e desaparecem quando se usa um passo suficientemente pequeno. Já nos casos $r=0$ e $r=0,50$ as soluções numéricas não oscilaram com o passo de 0,01.

Para cada valor de $r$, a velocidade de queda calculada por meio da série assintótica de (17) $\operatorname{com} n=100$ não oscilou.

Sendo $\theta_{0}$ o valor de $\theta$ para o qual a velocidade de queda é $99 \%$ da velocidade limite de Stokes, foi mostrado que os métodos numérico e assintótico produziram para cada caso o mesmo valor de $\theta_{0}$, onde

$$
\begin{aligned}
& \theta_{0}=7956,46(r=-0,25) \\
& \theta_{0}=6365,57(r=0) \\
& \theta_{0}=3183,79(r=0,50)
\end{aligned}
$$

Além disso, os resultados das Tabelas 1,2 e 3 mostram que, para cada $r$, os valores da velocidade de queda, a partir da convergência de cada série assintótica, obtidos pelos dois métodos são particularmente iguais.

Ainda de acordo com os dados das Tabelas 1,2 e 3, as séries assintóticas convergem para $\theta \geq 55,00(r=-0,25), \theta \geq 40(r=0)$ e $\theta \geq 25(r=0,50)$. Estes limites inferiores são bem menores que os respectivos valores de $\theta_{0}$ acima, mostrando que o método assintótico apresenta um largo alcance na direção de $\theta=0$, uma vez que $\theta$ é relativamente grande para $t$ pequeno.

\begin{tabular}{cccc}
\hline $\boldsymbol{\theta}$ & Runge-Kutta & Equação (30) & Série Assintótica \\
\hline $\mathbf{0 , 0 0}$ & 0,000000 & - & - \\
$\mathbf{0 , 5 0}$ & 0,225501 & $-0,262566$ & - \\
$\mathbf{1 , 0 0}$ & 0,336511 & 0,107938 & - \\
$\mathbf{1 , 5 0}$ & 0,409815 & 0,271634 & - \\
$\mathbf{2 , 0 0}$ & 0,463294 & 0,369217 & - \\
$\mathbf{2 , 5 0}$ & 0,504617 & 0,435810 & - \\
$\mathbf{3 , 0 0}$ & 0,537801 & 0,484968 & - \\
$\mathbf{3 , 5 0}$ & 0,565205 & 0,523173 & - \\
$\mathbf{4 , 0 0}$ & 0,588329 & 0,553969 & - \\
$\mathbf{4 , 5 0}$ & 0,608174 & 0,579478 & - \\
$\mathbf{5 , 0 0}$ & 0,625445 & 0,601058 & - \\
$\mathbf{1 0}, \mathbf{0 0}$ & 0,726091 & 0,717905 & - \\
$\mathbf{1 5 , 0 0}$ & 0,773976 & 0,769671 & - \\
$\mathbf{2 0}, \mathbf{0 0}$ & 0,802233 & 0,800529 & - \\
$\mathbf{2 5 , 0 0}$ & 0,822962 & 0,821588 & - \\
$\mathbf{3 0 , 0 0}$ & 0,838752 & 0,837133 & - \\
$\mathbf{3 5 , 0 0}$ & 0,850714 & 0,849214 & - \\
$\mathbf{4 0 , 0 0}$ & 0,860355 & 0,858953 & - \\
$\mathbf{4 5 , 0 0}$ & 0,868342 & 0,867019 & - \\
$\mathbf{5 0 , 0 0}$ & 0,875098 & 0,873843 & - \\
$\mathbf{5 5 , 0 0}$ & 0,880911 & 0,879714 & 0,880283 \\
$\mathbf{6 0 , 0 0}$ & 0,885981 & 0,884835 & 0,885333 \\
$\mathbf{6 5 , 0 0}$ & 0,890454 & 0,889353 & 0,889793 \\
\hline & & $1-V 010$ & \\
\hline
\end{tabular}

Tabela 1 - Velocidade de queda para $r=-0,25$ 


\begin{tabular}{cccc}
\hline $\boldsymbol{\theta}$ & Runge-Kutta & Equação (30) & Série Assintótica \\
\hline $\mathbf{0 , 0 0}$ & 0,000000 & - & - \\
$\mathbf{0 , 5 0}$ & 0,236329 & $-0,128379$ & - \\
$\mathbf{1 , 0 0}$ & 0,354070 & 0,202115 & - \\
$\mathbf{1}, \mathbf{5 0}$ & 0,431261 & 0,348530 & - \\
$\mathbf{2}, \mathbf{0 0}$ & 0,487036 & 0,435810 & - \\
$\mathbf{2}, \mathbf{5 0}$ & 0,529713 & 0,495373 & - \\
$\mathbf{3 , 0 0}$ & 0,563666 & 0,539341 & - \\
$\mathbf{3}, \mathbf{5 0}$ & 0,591463 & 0,573513 & - \\
$\mathbf{4 , 0 0}$ & 0,614730 & 0,601058 & - \\
$\mathbf{4 , 5 0}$ & 0,634552 & 0,623874 & - \\
$\mathbf{5 , 0 0}$ & 0,651688 & 0,643175 & - \\
$\mathbf{1 0 , 0 0}$ & 0,749468 & 0,747687 & - \\
$\mathbf{1 5}, \mathbf{0 0}$ & 0,794660 & 0,793987 & - \\
$\mathbf{2 0 , 0 0}$ & 0,821895 & 0,821588 & - \\
$\mathbf{2 5}, \mathbf{0 0}$ & 0,840591 & 0,840423 & - \\
$\mathbf{3 0 , 0 0}$ & 0,854455 & 0,854327 & - \\
$\mathbf{3 5 , 0 0}$ & 0,865240 & 0,865133 & 0,873902 \\
$\mathbf{4 0 , 0 0}$ & 0,873909 & 0,873843 & 0,881102 \\
$\mathbf{4 5 , 0 0}$ & 0,881080 & 0,881058 & 0,887196 \\
$\mathbf{5 0 , 0 0}$ & 0,887177 & 0,887162 & \\
\hline
\end{tabular}

Tabela 2 - Velocidade de queda para $r=0$

\begin{tabular}{cccc}
\hline $\boldsymbol{\theta}$ & Runge-Kutta & Equação (30) & Série Assintótica \\
\hline $\mathbf{0 , 0 0}$ & 0,000000 & - & - \\
$\mathbf{0 , 5 0}$ & 0,268193 & 0,202115 & - \\
$\mathbf{1 , 0 0}$ & 0,406742 & 0,435810 & - \\
$\mathbf{1 , 5 0}$ & 0,495729 & 0,539341 & - \\
$\mathbf{2 , 0 0}$ & 0,558098 & 0,601058 & - \\
$\mathbf{2}, \mathbf{5 0}$ & 0,604303 & 0,643175 & - \\
$\mathbf{3 , 0 0}$ & 0,639933 & 0,674265 & - \\
$\mathbf{3 , 5 0}$ & 0,668269 & 0,698428 & - \\
$\mathbf{4 , 0 0}$ & 0,691366 & 0,717905 & - \\
$\mathbf{4 , 5 0}$ & 0,710578 & 0,734038 & - \\
$\mathbf{5 , 0 0}$ & 0,726831 & 0,747687 & - \\
$\mathbf{1 0 , 0 0}$ & 0,813083 & 0,821588 & - \\
$\mathbf{1 5}, \mathbf{0 0}$ & 0,849568 & 0,854327 & - \\
$\mathbf{2 0 , 0 0}$ & 0,870724 & 0,873843 & 0,884905 \\
$\mathbf{2 5 , 0 0}$ & 0,884921 & 0,887162 & 0,895277 \\
$\mathbf{3 0 , 0 0}$ & 0,895285 & 0,896994 & 0,903272 \\
$\mathbf{3 5 , 0 0}$ & 0,903277 & 0,904635 & 0,909679 \\
$\mathbf{4 0 , 0 0}$ & 0,909682 & 0,910794 & 0,914961 \\
$\mathbf{4 5 , 0 0}$ & 0,914963 & 0,915896 & 0,919414 \\
$\mathbf{5 0 , 0 0}$ & 0,919415 & 0,920212 & \\
\hline
\end{tabular}

Tabela 3 - Velocidade de queda para $r=0,50$

Outro resultado interessante é a aproximação para a velocidade de queda dada por (30), para $\theta$ suficientemente grande. Ela produz os mesmos valores de $\theta_{0}$ já frisados e, por (30), serve como base para a obtenção de uma fórmula para $\theta_{0}$, caracterizada por

$\theta_{0}=(100 M)^{2}$, 


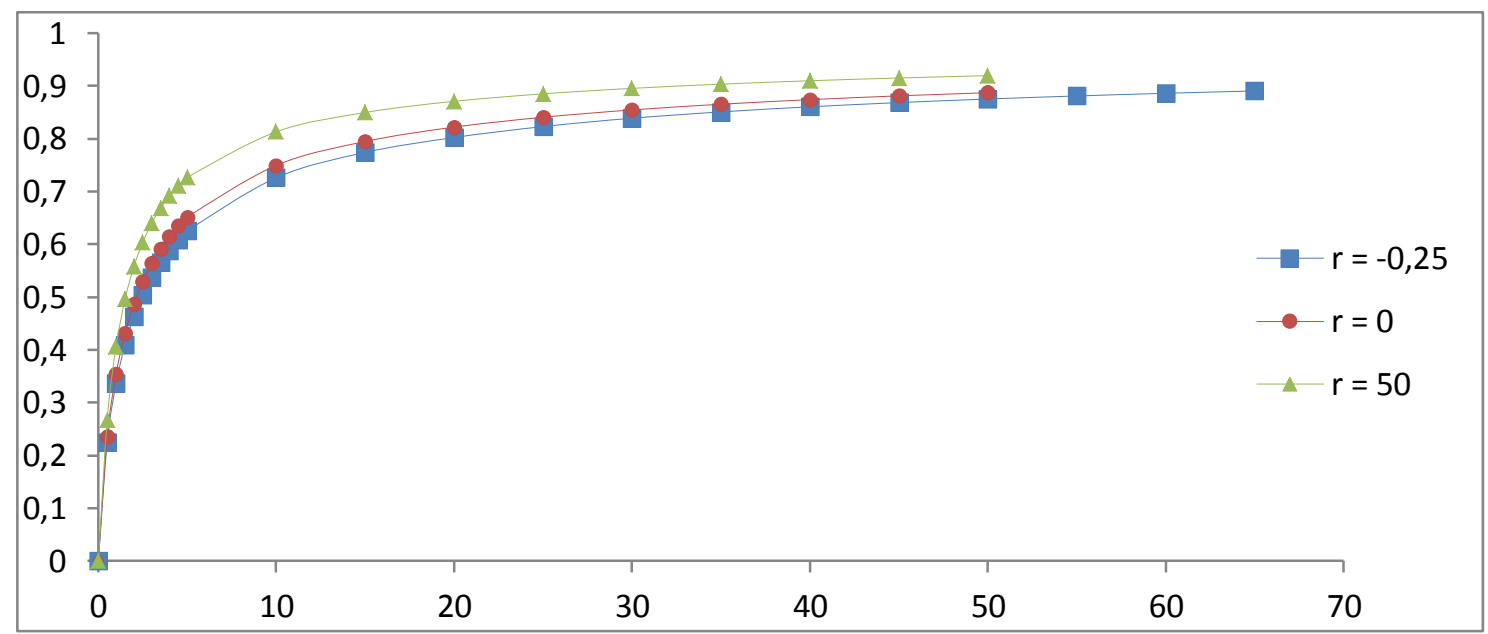

Figura 1 - Gráfico da velocidade de queda

Mas, não é só por isso, das Tabelas 1, 2 e 3 podemos ver que (30), para cada caso, também aproxima a velocidade de queda para valores bem menores que $\theta_{0}$. Ou seja, pelas tabelas, as velocidades são aproximadamente dadas por (30) para $\theta \geq 15(r=-0,25)$, $\theta \geq 10(r=0)$ e $\theta \geq 15(r=0,50)$, tendo, para cada $r$, a relação (30) um alcance maior na direção $\theta=0$ que a série assintótica $\operatorname{com} n=100$.

\section{Conclusões}

A velocidade de queda é limitada e crescente, aproximando-se assintoticamente da velocidade limite de Stokes, como mostram as curvas do gráfico apresentado pela Figura 1.

A forma do problema (12) e (13) realmente sugere caminhos para a obtenção da velocidade de queda. Ela, além da série assintótica, evidencia a aproximação (30) por substituição direta de (30) em (12) e (13).

A transformação da Equação íntegro-diferencial em equação diferencial abre caminho para estudos de quedas de corpos em fluidos que requerem termos adicionais, no sentido de que, para não envolver história de aceleração, os termos adicionais sejam colocados na Equação (12).

\section{Referências Bibliográficas}

ARDERKANI, A. M; RANGEL, R, H. Unsteady motion of two solid spheres in Stokes flow. Physics of Fluids. v.18, 2006.

BASSET, A. B. A Treatise on Hydrodynamics. Cambridge: Daighton Bell, 1961, v. 2.

FENG, J; JOSEPH, D. D. The unsteady motion of solid bodies in creeping flows. Journal of Fluid Mechanics. v. 303, p. 83-102, 1995.

MOLLINGER, A. M; CORNELISSEN, E. C; VAN DEN BRULE B. H. A. A. An unexpected phenomenon observed in particle settling: oscillating falling spheres. Journal of Non-Newtonian Fluid Mechanics. v. 86, p. 389-393, 1999.

SOARES, A. A; CARAMELO, L; ANDRADE, M. A. P. M. Study of the motion of a vertically falling sphere in a viscous fluid. European Journal of Physics. v.33, p. 1053-1062, 2012.

SRIVATAVA, D, K; SRIVASTAVA, N. Stokes flow past axially symmetric isolated bodies: A review article. Journal of Applied Mathematics and Mechanics. v. 9, p. 1-53, 2013. 